\title{
Selective internal radiation therapy: an effective treatment for hormonal syndromes in pancreatic neuroendocrine tumors
}

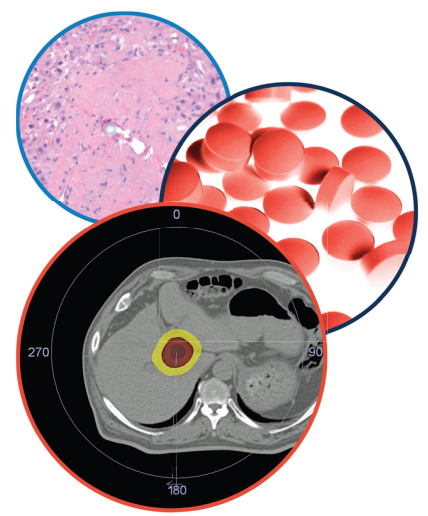

Hepatic Oncology

\author{
Leyre Zubiri*,1, José I Bilbao², Javier Rodríguez ${ }^{3}$ \& Bruno Sangro4 \\ ${ }^{1}$ Medical Oncology, Clínica Universidad de Navarra, Pamplona, Spain \\ ${ }^{2}$ Interventional Radiology, Clínica Universidad de Navarra, Pamplona, Spain \\ ${ }^{3}$ Medical Oncology, Clínica Universidad de Navarra, Pamplona, Spain \\ ${ }^{4}$ Liver Unit (HPB Oncology Area), Clínica Universidad de Navarra, Pamplona, Spain \\ *Author for correspondence: Tel.: +34 676263 015; iriazubi11@hotmail.com
}

The hormone secretion in pancreatic neuroendocrine tumors ( $p N E T$ ) causes an important interference in patients' quality of life. We present two cases of pNET metastatic to the liver (a pancreatic endocrine carcinoma with a severe hormonal syndrome and an insulinoma with severe crisis of hypoglycemia and coma) refractory to conventional treatments, which were finally solved with selective internal radiation therapy (SIRT), a nonstandard level 1 therapy. We show two examples of an excellent control of symptoms together with a long survival after treatment with SIRT. The evidence supporting the use of this therapy is level 2. Our case reports strongly support the use of SIRT for the severe clinical syndrome in pNET metastatic to the liver and refractory to somatostatin analogs.

First draft submitted: 5 December 2017; Accepted for publication: 13 June 2018; Published online: 28 September 2018

Keywords: hormonal syndrome • neuroendocrine tumors • selective internal radiation therapy

\section{Practice points}

- Pancreatic neuroendocrine tumors (pNETs) are increasing in incidence in most countries and therefore require further attention.

- There are two problems to deal with when treating pNETs: the challenge of achieving an antitumoral response, and the treatment of hormone-excess states, which can be really harmful and cause a life-long problem.

- The cornerstone of treatment is surgery with curative intent, directed to the eradication of tumor and the alleviation of hormonal syndrome. However, surgery is not possible in many cases, due to the spread of disease.

- The standard therapy in functioning PNET is the use of somatostatin analogs (SSA). If progression on first line with SSA develops, there is not a unique defined standard approach and the optimal management strategy is uncertain.

- Historically, cytotoxic therapies and interferon were used to treat refractory disease, but the action was too slow. Another available option in PNET with hepatic lesions is the use of liver-therapies, as most of the lesions are hypervascular and can be treated with local procedures (transarterial embolization, surgery and ablative therapy).

- In recent years, different new systemic therapies have been developed including everolimus and sunitinib, with proven patient benefit in Phase III trials with relevant end points, and, more recently, chemotherapy with dacarbazine or capecitabine doublets or combinations of bevacizumab with octreotide or everolimus have shown promising results in terms of response and survival.

- In many cases, however, the achievement of an antitumoral response either with these systemic agents or with liver-directed therapies does not translate into a control of the hormone-excess state. Finally, the fact that symptom control is not frequently the primary end point in studies makes it difficult to reach a solid consensus.

Pancreatic neuroendocrine tumors (pNETs) have shown an increasing incidence over the last 30 years [1,2], for reasons that are largely unknown. While most pNETs are non-functional, 10-30\% of them present with symptoms due to hormone secretion (functional tumors) [3]. Among the tumors that produce a hormonal syndrome, 70\% are insulinomas and $25 \%$ are a mix of glucagonomas, gastrinomas and somatostatinomas. Pancreatic endocrine 
carcinomas (VIPomas) and other rare entities represent only $5 \%$ of all pNET. The prognosis of pNETs when they are metastatic is poor, with a median overall survival of 24 months [4].

The consequences of hormone secretion in metastatic pNET can be really disabling and cause an important interference in patients' quality of life. Several studies have shown that hormone-excess states are frequent causes of death if they are not adequately treated [5]. Control of symptoms from hormonal hypersecretion in the advanced disease is often urgent and somatostatin analogs (SSA) are the standard therapy in functioning pNETs. SSA are highly effective in symptom control [6] and, furthermore, may prolong progression-free survival [7].

If symptomatic refractory disease develops in spite of treatment with SSA, other therapies can be attempted. Cytoreductive surgery may improve symptomatic control and impact in survival. It should be considered in the presence of localized metastatic disease or when most tumor load (>90\%) can be resected [8]. Yet, only $20 \%$ of patients are candidates for surgical treatment [9]. Transarterial embolization and chemoembolization have shown efficacy in symptomatic control, [10] but their effects are short, and the correct approach and optimal sequence of these therapies remain controversial [11,12]. Everolimus and peptide receptor radionuclide therapy (PRRT; after confirming the presence of expression of SSTR2 by somatostatin receptor imaging) are effective in treatment of hypoglycemia in metastatic insulinoma and can be used after failure to SSA [13], although attention should be paid in patients with insulinoma, where inhibition of insulin by SSA is weak, due to a minor expression of somatostatin receptors in these tumors. As a result, this might in turn provoke the inhibition of competitive hormones such as glucagon, leading to an aggravation of the hypoglycemia [14].

Selective internal radiation therapy (SIRT), also called radioembolization, consists in the delivery of $\beta$ radiation to liver tumors through the injection of radioactive microspheres into the hepatic artery or its branches. It has been reported to be an effective therapy that induces durable responses and produces encouraging progression-free survival as well as symptomatic relief [15]. Most of the reported series are cohort studies or Phase II trials, and prospective studies assessing the effect on syndrome control are scarce $[9,16]$.

Here, we present two patients with severe hormonal syndrome due to insulinoma and VIPoma metastatic to the liver, refractory to conventional treatments, which finally achieved a complete resolution of the hormonal syndrome after treatment with SIRT.

\section{Presentation of cases \\ Case 1}

A 50-year-old woman who had presented with a history of diarrhea and a $15 \mathrm{~kg}$ weight loss had a diagnosis of VIPoma metastatic to the liver. Serum VIP and $24 \mathrm{~h}$ urine 5-hydroxyindoleacetic acid levels were elevated at $75 \mathrm{pmol} / \mathrm{l}$ and $25 \mathrm{mg} / 24 \mathrm{~h}$, respectively. The primary tumor was removed by a distal pancreatectomy and inmunohistochemical analysis of the surgical specimen showed positivity for cromogranin, sinaptofisin and pancreatic polypeptide. Unfortunately, Ki67 data, which is extremely important in this type of tumor, was not available. After surgery, the primary goal was to rescue the patient for resection by downstaging with a treatment that could produce an objective remission of the disease and allow a two-stage surgical approach, so combination of transarterial embolization (TAE) plus chemotherapy was chosen for this purpose. Chemotherapy with 5-fluorouracil (5-FU) and streptozotocin was then administered in combination with transarterial bland embolization for 9 months. Several embolizations of tumoral arteries with 150-200 $\mu \mathrm{m}$ polyvinyl alcohol particles (PVA) were performed and resulted in minor radiological response by a computerized tomograph scan but complete symptomatic control. These repeated sessions of TAE led to 1 year of stable disease in the liver and no extrahepatic metastases. A radical two-stage surgical approach was then planned, but it was finally dismissed due to an expected small future liver remnant. 9 months later, she developed persistent watery diarrhea, lost $10 \mathrm{~kg}$ of weight and had severe electrolyte disturbances (metabolic acidosis with serum potassium of $1.9 \mathrm{mEq} / \mathrm{l}$ ). Long-acting octreotide was initiated at a dose of $30 \mathrm{mg}$ every 4 weeks with only a partial and transient symptomatic amelioration. When severe watery diarrhea and electrolyte disturbances reappeared, unilobar SIRT was uneventfully performed. The procedure targeted the right hepatic lobe. SIRT-related toxicity was not observed. After the procedure, the patient decided to stop longacting octreotide and remained asymptomatic for the following 2 years. Cromogranin A levels dropped from 32 $\mathrm{U} / \mathrm{I}$ to normal and remained normal during that time. Image tests (magnetic resonance imaging and computerized tomography) showed barely any decrease in size, but a decrease in intralesional vascularity that conferred tumors a ring enhancement in the arterial phase. Diarrhea, weight loss and metabolic acidosis recurred and octreotide was then resumed. (Figure $1 \mathrm{~A}-\mathrm{D}$ ). 


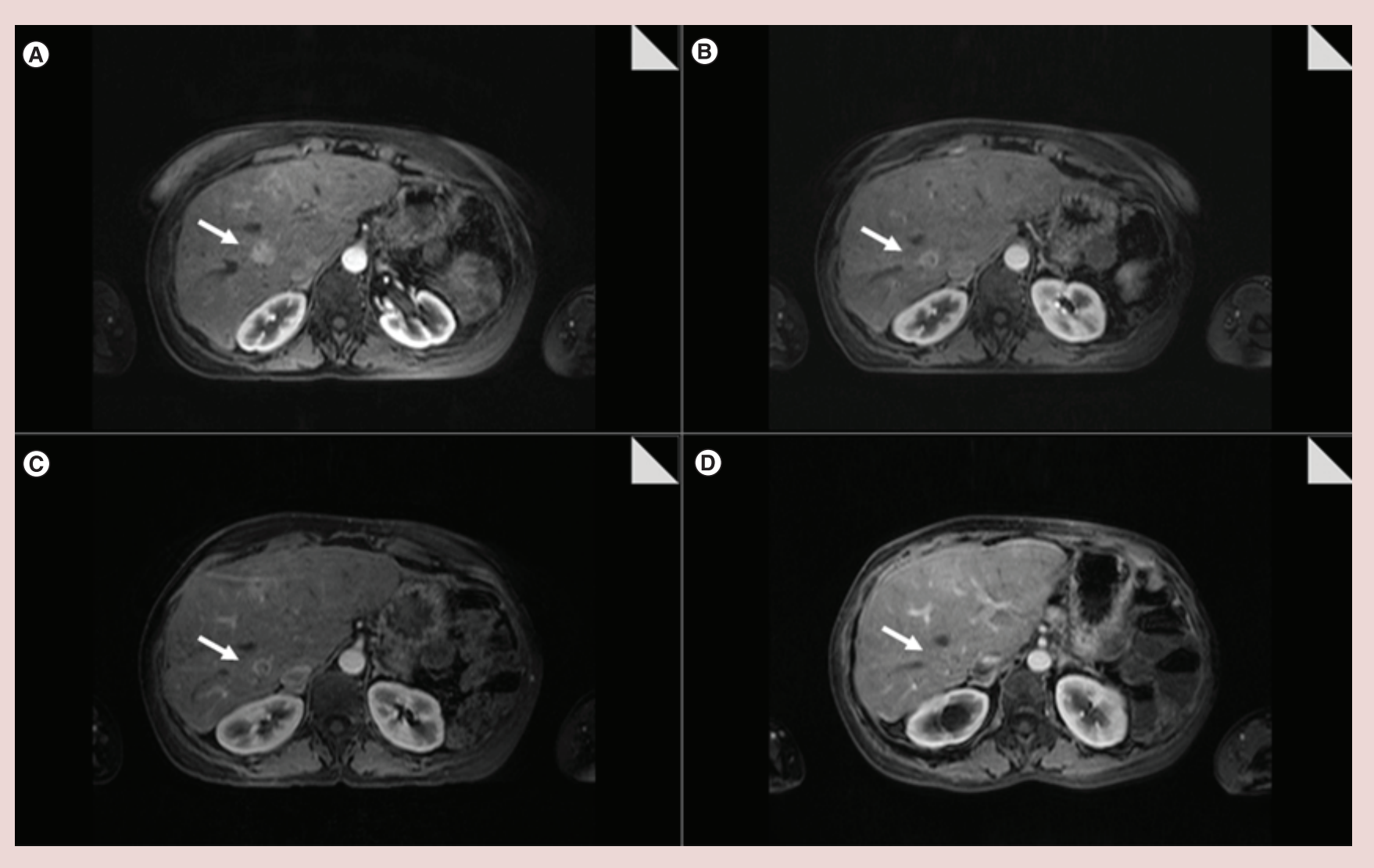

Figure 1. Magnetic resonance imaging images of VIPoma lesions at different moments of the disease. (A) Basal. (B) 6 months after selective internal radiation therapy. (C) 12 months after selective internal radiation therapy. (D) 2 years after selective internal radiation therapy. A ring enhancement can be observed in the tumor, secondary to a decrease in intralesional vascularity.

\section{Case 2}

A 68-year-old man developed severe hypoglycemia and had a diagnosis of pancreatic insulinoma that was treated with a caudal pancreatectomy. Immunohistochemical analysis of the surgical specimen was positive for insulin. We did not have Ki 67 data available either in this patient, an element of absolute importance that would have been of high interest in both cases. Liver surgery for resection of isolated liver metastases in segments IVb and III was performed 2 and 5 years later, respectively. Symptomatic liver progression with multiple unresectable liver metastases occurred 1 year later. As long-acting octreotide was not available at that time, chemotherapy with Streptozotozine and 5-FU was selected in an attempt to avoid octreotide injections three-times daily in case of efficacy. New hepatic lesions (segments II, VII and VIII) were observed after three courses of chemotherapy, and treatment with Sandostatin was then started at a dose of $50 \mathrm{mcg} / 8 \mathrm{~h}$ and later increased to a dose of $100 \mathrm{mcg} / 8 \mathrm{~h}$. Symptom control was achieved with treatment with octreotide but severe crisis recurred after 1 year. Three transarterial bland embolization procedures were performed using 150-300 $\mu \mathrm{m}$ and 300-500 $\mu \mathrm{m}$ PVA particles, but proved unsuccessful in achieving long-lasting symptomatic control. He finally had to be admitted to the intensive care unit because he needed a continuous intravenous perfusion of octreotide and glucose to avoid severe crisis of hypoglucemia leading to coma. A new embolization procedure (left hepatic artery with 150-300 $\mu \mathrm{m}$ PVA particles) provided no benefit and was complicated by upper gastrointestinal bleeding. A total of 2 weeks later, after having completed repeated sessions of TAE with no response, SIRT was performed. The procedure targeted the right hepatic artery. After 4 days, intravenous perfusions could be interrupted and the patient was discharged, asymptomatic under subcutaneous octreotide ( $300 \mathrm{mcg}$ daily). A total of 2 months after SIRT, transient, mild hypertransaminasemia was observed. There was no evidence of other SIRT-related side effects. Consecutive computerized tomography scans showed a progressive shrinkage of all liver lesions until complete response was recorded after 8 months. He never suffered from hypoglycemia thereafter but was kept on long-acting octreotide 


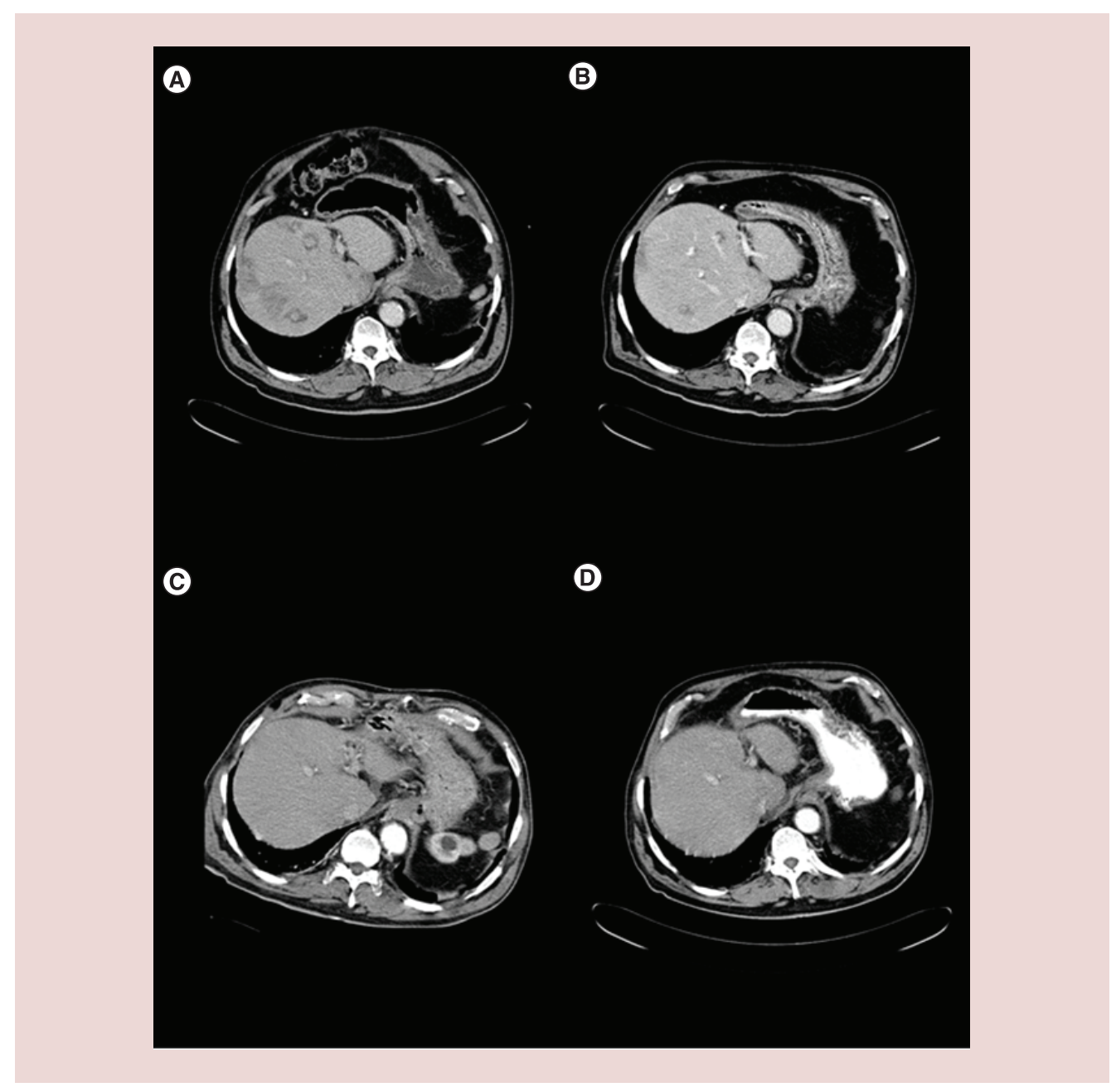

Figure 2. Computerized tomography scan images of insulinoma lesions at different moments of the disease. (A) Basal. (B) 3 months after selective internal radiation therapy. (C) 6 months after selective internal radiation therapy. (D) A year after selective internal radiation therapy. A complete response is shown with no evidence of tumoral lesions. Concurrently with the radiological response, the hormonal syndrome disappeared.

permanently. A total of 11 months after SIRT, he had a severe and complicated thromboembolic episode consisting of brain stroke, bilateral femoral thrombosis and severe pulmonary embolism. He died 17 months later while in complete response of his liver metastatic tumor (Figure $2 \mathrm{~A}-\mathrm{D}$ ).

\section{Discussion}

Pancreatic neuroendocrine tumors are rare tumors and their natural history is quite different from the more common pancreatic adenocarcinoma. Almost $40 \%$ of patients develop metastases, most frequently in the liver [8], and can potentially suffer from a severe clinical syndrome related to hormone secretion. Therefore, an optimal approach with an effective treatment that may help achieving symptomatic control is essential.

SSAs must be used in patients with functional pNET and distant metastases prior to other specific antitumor treatments (surgical or locoregional), as they provide symptom alleviation together with a biochemical response [17]. However, symptoms may not be fully or persistently controlled despite optimal SSA therapy, due to an as of yet unresolved escape phenomenon [18]. SSA induce an important symptomatic improvement in most of the cases (70\%), [19] but patients usually become refractory to this therapy because of the development of desensitization [20] months after the beginning of the treatment [21].

Liver-directed therapies including surgery, percutaneous ablation and transarterial procedures are used to treat pNET metastatic to the liver with the aim of controlling symptoms and improving patient survival $[10,11,22]$. Our two 
cases show how resection and bland embolization can be used for this purpose. Nevertheless, the efficacy of resection, ablation and embolization is limited by the number and location of the tumors. Patients with widespread liver disease are not amenable to these therapies and embolizing therapies may usually have a short-lasting effect $[8,15]$. Furthermore, the best sequence for performing these procedures is not well established considering the high variability in the rate and pattern of progression of these tumors.

SIRT aims to deliver significant amounts of $\beta$ radiation to liver tumors irrespective of their size, number and location [23]. Radioactive particles are injected into the hepatic artery or its branches and then depend on the arterial blood flow to reach their target deposition in the tumor vasculature [24]. pNET metastases are very highly vascularized liver tumors. This results in a high tumor to nontumor distribution of the microspheres that allows delivery of a high amount of radiation to liver metastases. This in turn results in high response rates to SIRT with the alleviation of symptoms and prolonged progression-free and overall survival [25].

Chemotherapy has been classically used for growth and symptomatic control in pNET, but its slow duration of action makes it not the best option to treat the hormone excess state in its acute onset. With the lack of a consistently effective chemotherapy for pNET patients [26] with severe clinical syndrome [27], SIRT has shown promising results in the control, eradication or simply debulking of hepatic metastases, while it may also be used to alleviate carcinoid syndrome or mechanical symptoms such as pain and obstruction [15]. The evidence supporting the use of SIRT is level 2 and there is only one prospective study $(n=34)$ that shows syndrome control (55\% response) [28]. SIRT is listed in guidelines among the therapies that can be used to treat pNET tumors metastatic to the liver [29,30], although further investigations are needed to define the exact role of SIRT and its effect in biomarker response, local antitumoral effect, symptomatic control, and survival, as it is still considered investigational.

SIRT offers several advantages over bland embolization or chemoembolization for the treatment of liver tumors. It causes a postembolization syndrome that is less frequent and milder [15,31], resulting in better postprocedural quality of life [32], need not be repeated, and can be offered as an outpatient procedure [11]. Thus, SIRT provides better tolerability for a procedure that is at least equally effective to, if not better than, other embolizing techniques. Although systemic therapies like everolimus can alleviate hormone syndromes [26,27], and treatments with radiolabeled SSA like PRRT have demonstrated morphological, biochemical, and clinical responses [33], SIRT may be an as good as, if not a better than, option for patients with liver-only disease because of the rapid onset of its beneficial effect as illustrated by case 2 . The two cases we have reported here support the consideration of SIRT as an alternative to treat the severe clinical syndrome in pNET metastatic to the liver when somatostatin analogs are not able to provide adequate symptom control, although further randomized studies are needed to determine its exact role and its possible combination with other therapies like PRRT, either using SIRT as a salvage treatment option after PRRT failure [34] or as a sequential strategy in the treatment algorithm [35]. Finally, these cases might endorse the convenience of conducting cost-effective analysis, since the cost of prolonged use of somatostatin analogs cannot be ignored.

\section{Future perspective}

Apart from the secondary disability due to hormone syndrome, the presence of liver disease in patients with pNET is one of the factors that confer a worst prognosis. Hepatic intra-arterial therapies (including TAE, TACE and SIRT) have demonstrated similar results in response, tumor growth, symptomatic control and survival but a lower incidence of side effects in favor of SIRT. In addition, SIRT often requires a single treatment, in contrast to TAE/TACE, which frequently has to be repeated several times. Moving forward, it is reasonable to think that SIRT could replace TACE/TAE in many cases. Nevertheless, evidence is scarce as SIRT has emerged as a new therapy over the past twenty years and further controlled trials are needed to investigate the superiority of one treatment over the other.

\section{Financial \& competing interests disclosure}

JIB, JR and BS have received advisory and/or lecture fees from Sirtex Medical; BS has received advisory fees from BTG. The authors have no other relevant affiliations or financial involvement with any organization or entity with a financial interest in or financial conflict with the subject matter or materials discussed in the manuscript. This includes employment, consultancies, honoraria, stock ownership or options, expert testimony, grants or patents received or pending, or royalties.

No writing assistance was utilized in the production of this manuscript. 


\section{Ethical conduct of research}

The authors state that for investigations involving human subjects, informed consent has been obtained from the participants involved.

Additional informed consent was obtained from all individual participants for whom identifying information is included in this article.

\section{Open access}

This work is licensed under the Attribution-NonCommercial-NoDerivatives 4.0 Unported License. To view a copy of this license, visit http://creativecommons.org/licenses/by-nc-nd/4.0/

\section{References}

Papers of special note have been highlighted as: $\bullet$ of interest or $\bullet \bullet$ of considerable interest

1 Fraenkel M, Kim M, Faggiano A, Herder WW, Valk GD. Incidence of gastroenteropancreatic neuroendocrine tumours: a systematic review of the literature. Endocr. Relat. Cancer 21(3), 153-163 (2014).

2 Hallet J, Law CH, Cukier M, Saskin R, Liu N, Singh S. Exploring the rising incidence of neuroendocrine tumors: a population-based analysis of epidemiology, metastatic presentation, and outcomes. Cancer 121(4), 589-597 (2015).

3 Young K, Iyer R, Morganstein D, Chau I, Cunningham D, Starling N. Pancreatic neuroendocrine tumors: a review. Future Oncol. 11(5), 853-864 (2015).

4 Kunz PL. Carcinoid and neuroendocrine tumors: building on success. J. Clin. Oncol. 33(16), 1855-1863 (2015).

5 Ito T, Igarashi H, Jensen RT. Pancreatic neuroendocrine tumors: clinical features, diagnosis and medical treatment: advances. Best Pract. Res. Clin. Gastroenterol. 26(6), 737-753 (2012).

6 Kulke MH, Shah MH, Benson AB 3rd et al. Neuroendocrine tumors, version 1.2015. J. Natl Compr. Canc. Netw. 13(1), 78-108 (2015).

7 Caplin ME, Pavel M, Ćwikła JB et al. Lanreotide in metastatic enteropancreatic neuroendocrine tumors. N. Engl. J. Med. 371(3), 224-233 (2014).

- Confirms an antitumoral response of somatostatin analogs on enteropancreatic neuroendocrine tumors, apart from the known effect in symptomatic control due to hormonal syndrome.

8 Rossi RE, Massironi S, Conte D, Peracchi M. Therapy for metastatic pancreatic neuroendocrine tumors. Ann. Transl. Med. 2(1), 8 (2014).

9 Clift AK, Frilling A. Management of patients with hepatic metastases from neuroendocrine tumors. Ann. Saudi Med. 34(4), 279-290 (2014).

10 Frilling A, Clift AK. Therapeutic strategies for neuroendocrine liver metastases. Cancer 121(8), 1172-1186 (2015).

11 Kennedy A, Bester L, Salem R, Sharma RA, Parks RW, Ruszniewski P. Role of hepatic intra-arterial therapies in metastatic neuroendocrine tumours (NET): guidelines from the NET-liver-metastases consensus conference. HPB (Oxford) 17(1), 29-37 (2015).

12 Del Prete M, Fiore F, Modica R et al. Hepatic arterial embolization in patients with neuroendocrine tumors. J. Exp. Clin. Cancer Res. 33, 43 (2014).

13 Bernard V, Lombard-Bohas C, Taquet MC et al. Efficacy of everolimus in patients with metastatic insulinoma and refractory hypoglycemia. Eur. J. Endocrinol. 168(5), 665-674 (2013).

14 Igarashi H, Hijioka M, Lee L, Ito T. Biotherapy of pancreatic neuroendocrine tumors using somatostatin analogs. J. Hepatobiliary Pancreat. Sci. 22, 618-622 (2015).

15 Paprottka PM, Hoffman RT, Haug A et al. Radioembolization of symptomatic, unresectable neuroendocrine hepatic metastases using yttrium-90 microspheres. Cardiovasc. Intervent. Radiol. 35(2), 334-342 (2012).

-. An analysis of the role of radioembolization on unresectable neuroendocrine tumors metastatic to the liver when refractory to other treatments. It shows its effect on tumor response, tumor-marker levels and symptomatic control.

16 Devcic Z, Rosenberg J, Braat AJ et al. The efficacy of hepatic 90Y resin radioembolization for metastatic neuroendocrine tumors: a meta-analysis. J. Nucl. Med. 55(9), 1404-1410 (2014).

17 Raj N, Reidy-Lagunes D. Systemic therapies for advanced pancreatic neuroendocrine tumors. Hematol. Oncol. Clin. North Am. 30(1), 119-133 (2016).

18 Verbeek, WH M, Korse CM, Tesselaar ME. Secreting gastro-enteropancreatic neuroendocrine tumours and biomarkers. Eur. J. Endocrinol. 174(1), R1-R7 (2016).

19 Wolin EM, Malon A, Chassaing C et al. Lanreotide depot: an antineoplastic treatment of carcinoid or neuroendocrine tumors. J. Gastrointest. Cancer 47(4), 366-374 (2016).

20 Orlewska E, Bednarczuk T, Kaminski G, Kos-Kudla B. LanroNET, a non-interventional, prospective study to assess the resource utilization and cost of lanreotide autogel $120 \mathrm{mg}$ in polish patients with neuroendocrine tumors: results of interim analysis. Contemp. Oncol. 18(6), 442-447 (2014). 
21 Paragliola RM, Prete A, Papi G et al. Clinical utility of lanreotide autogel ${ }^{\circledR}$ in gastroenteropancreatic neuroendocrine tumors. Drug Des. Devel. Ther. 10, 3459-3470 (2016).

22 Grandhi MS, Lafaro KJ, Pawlik TM. Role of locoregional and systemic approaches for the treatment of patients with metastatic neuroendocrine tumors. J. Gastrointest. Surg. 19(12), 2273-2282 (2015).

23 Tong AK, Kao YH, Too CW, Chin KF, Ng DC, Chow PK. Yttrium-90 hepatic radioembolization: clinical review and current techniques in interventional radiology and personalized dosimetry. Br. J. Radiol. 89(1062), 20150943, (2016).

- A review article that analyses the effects of yttrium-90 ([90]Y) microsphere radioembolization in terms of efficacy for primary and metastatic liver malignancies, giving prominence to its safety profile and its consequent expanded use.

24 Kuei A, Saab S, Cho SK, Kee ST, Lee EW. Effects of Yttrium-90 selective internal radiation therapy on non-conventional liver tumors. World J. Gastroenterol. 21(27), 8271-8283 (2015).

25 Padia SA, Lewandowski RJ, Johnson GE et al. Radioembolization of hepatic malignancies: background, quality improvement guidelines, and future directions. J. Vasc. Interv. Radiol. 28(1), 1-15 (2017).

- An update of radioembolization therapy for primary and secondary liver tumors including colorectal cancer metastases, metastatic neuroendocrine tumors and other neoplasias, emphasizing its role in improving tumor responses and survival and minimizing adverse events.

26 Cives M, Strosberg J. Treatment strategies for metastatic neuroendocrine tumors of the gastrointestinal tract. Curr. Treat. Options Oncol. 18(3), 14 (2017).

27 Ito T, Lee L, Jensen RT. Treatment of symptomatic neuroendocrine tumor syndrome. Expert. Opin. Pharmacother. 17(16), 2192-2205 (2016).

28 King J, Quinn R, Glenn DM et al. Radioembolization with selective internal radiation microspheres for neuroendocrine liver metastases. Cancer 113(5), 921-929 (2008).

-. Prospective study that assess the safety and efficacy of selective internal radiation therapy in patients with inoperable liver metastases showing promising results with long-term responses.

29 Kennedy A, Coldwell D, Sangro B, Wasan H, Salem R. Integrating radioembolization into the treatment paradigm for metastatic neuroendocrine tumors in the liver. Am. J. Clin. Oncol. 35(1), 393-398 (2012).

30 Pavel M, O’Toole D, Costa F et al. ENETS consensus guidelines update for the management of distant metastatic disease of intestinal, pancreatic, bronchial neuroendocrine neoplasms (NEN) and NEN of unknown primary site. Neuroendocrinology 103(2), 172-185 (2016).

31 Abdel-Rahman OM, Elsayed Z. Yttrium-90 microsphere radioembolisation for unresectable hepatocellular carcinoma. Cochrane Database Syst. 2, 11-15 (2016).

32 De Baere T, Deschamps F, Tselikas L et al. GEP-NETs update: interventional radiology: role in the treatment of liver metastases from GEP-NETs. Eur. J. Endocrinol. 172(4), R151-R166 (2015).

- A review about different ablation liver therapies in oligometastatic patients diagnosed with neuroendocrine tumors and its effectiveness and safety in comparison with toxicity induced by systemic treatments.

33 Oronsky B, Ma PC, Morgensztern D et al. Nothing but NET: a review of neuroendocrine tumors and carcinomas. Neoplasia 19(12), 991-1002 (2017).

34 Ezziddin S, Meyer C, Kahancova S et al. 90Y Radioembolization after radiation exposure from peptide receptor radionuclide therapy. $J$. Nucl. Med. 53(11), 1663-1669 (2012).

35 Flippi L, Ciorra A, Sardella B et al. Sequential use of (90)Y microspheres radioembolization and (177)Lu-dotatate in pluri-metastatic neuroendocrine tumors: a case report. Nucl. Med. Mol. Imaging 48(4), 321-325 (2014). 
